\title{
Letter to the Editor: Outcome of Self-Expanding Metal Stents in the Treatment of Anastomotic Leaks After Ivor Lewis Esophagectomy
}

\author{
Victor D. Plat ${ }^{1} \cdot$ Freek Daams $^{1} \cdot$ Donald L. van $\operatorname{der}^{1}$ Pet $^{1}$
}

Published online: 20 May 2019

(C) Société Internationale de Chirurgie 2019

With great interest, we read the retrospective case series by Plum et al. [1] regarding the management of esophageal anastomotic leakage. Authors reported their experience with self-expanding metal stents (SEMS) in the management of anastomotic leakage following Ivor Lewis esophagectomy. Postoperative leakage was managed successfully in 49 out of 70 patients. After reading the results of their study, we would like them to address the questions and remarks we put forward.

In the present article, a differentiation was made between technical success $(71.4 \%)$ and treatment success (70\%). Can the authors elaborate on how this distinction may have led to a discrepancy in the percentages between the two definitions of success?

The authors defined treatment success as a visualized closure of the defect upon endoscopic stent removal. Treatment failure was defined as persistent leakage/fistula, death or the need for surgical revision. Interestingly, Table 3 shows one patient suffering from a gastrointestinal fistula in the successful cohort, which seems contradictory to the author's definition of success. As no routine CT imaging or contrast esophagograms were performed, do the authors think that such complications might have been missed? Do they believe that routine monitoring for these complications can be omitted?

As great variations in their management of leakage (indications for stent placement, type of stents and post- treatment protocol) are reported, we would like the authors to elaborate on how these successful results can be obtained by others?

Finally, the extension of leakage was briefly described. The defects were located directly at the anastomosis or along the longitudinal staple line, ranging from one-fourth to half of the circumference of the esophagus. We wonder whether the authors have taken the presence of ischemia, necrosis and dilatation of the gastric conduit into account. As the extent of anastomotic leakage and choice of treatment is not solely based on the size of the defect [2].

In conclusion, we want to congratulate Plum and coauthors on their clinically relevant publication and look forward to their comments.

\section{References}

1. Plum PS, Herbold T, Berlth F et al (2019) Outcome of selfexpanding metal stents in the treatment of anastomotic leaks after Ivor Lewis esophagectomy. World J Surg 43(3):862-869

2. Scheepers JJ, van der Peet DL, Veenhof AA et al (2010) Systematic approach of postoperative gastric conduit complications after esophageal resection. Dis Esophagus 23(2):117-121

Publisher's Note Springer Nature remains neutral with regard to jurisdictional claims in published maps and institutional affiliations.
Victor D. Plat

V.plat@vumc.nl

1 Department of Gastrointestinal Surgery, VU University Medical Center, Amsterdam UMC, Amsterdam, The Netherlands 